\title{
The role of learning technology in planning change in curriculum delivery and design
}

\author{
Colin Harrison
}

\author{
Anglia Polytechnic University, UK
}

\begin{abstract}
This paper is a discussion of some aspects of the application of learning technology to the design and delivery of flexible learning packages, which can help make the most effective use of the most valuable resource in a university - its staff. Principles of good course design, preparation and use are exemplified in the approach taken towards flexible learning packages at Anglia Polytechnic University.
\end{abstract}

\section{Introduction}

Learning technology is not chiefly concerned with using computers, video recorders, audio tapes or any other form of technology. Rather, it is concerned with a systematic approach to the planning and delivery of the educational experience. In other words, it should be more concerned with the design of the whole learning experience than with any particular delivery medium. From good design it should become clear how best to deliver a quality learning experience. This paper will therefore not be about the technology but more about the process and the initiatives that we in higher education are launching to share that planning and delivery process.

But since the principles of leaming technology have been around for many years, why suddenly bring it back to the front burner and ask that colleagues reassess its place in strategies for teaching and learning? Simply because it offers us a flexible model that will aid us not only in coping with the increase in student numbers but will also improve the learning experience of students. The pressures that have led us to look at new (or not so new) ways of designing the learning experience can, at one level, be said to stem from the major growth in higher education encouraged by the government in the 1990s. The target then set, of increasing the participation rate of 18-19 year-olds from one in five to one in three by the year 2000 , was used by the universities as a pathway to a rapid growth and a way of increasing income. The year 2000 target is already in sight and we have a slow-down imposed on us. We have 
also seen a jump in the numbers of mature students in higher education, a $70 \%$ increase in the 10 years up to 1990 . These two factors - more students leading to greater staff-student ratios, and an increase in the mature mix - has forced all universities to look at ways of planning their educational programmes to preserve the best experience, to provide a flexible pattern of attendance for mature students and to allow staff to survive all the pressures.

Against this background of increases in participation in higher education, and our desire to maintain quality and widen access, particularly within the wider region that many of the new universities in the UK serve, we face the pressure of having to deliver even further efficiency gains; and all this without increased resources for the university or our students. So a rethink is called for in how we make the most effective use of the most costly resource in the university - the staff. We need to adopt new approaches to teaching and learning.

There are also positive, pedagogic, reasons for change. There is real evidence that more independent study, flexibility of pace and place, and the appropriate use of technology can produce better results and enhance the transferability of intellectual and personal skills that is sought by employers. It allows for greater access via franchises to regional colleges, it supports mature students, and it allows more options to run each year regardless of student numbers.

All the evidence is that in facing up to these pressures for change we (as a sector) seem to be moving in three distinct stages. These have been defined by Wagner (1993) as follows:

- A first stage is usually more intensive use of existing methods resulting in classes getting larger, options reduced, the hours that students are taught being reduced and the hours that staff teach increased.

- The second stage is change but not fundamental change. For example it is realized that seminar groups of 25 begin to challenge the fundamental purpose of seminars. So, some rethinking occurs as to how to make the most cost effective use of both the most valuable and most costly resource in higher education - the time of an academic. This problem has been characterized as the division between 'mass time' and 'quality time'. In higher education the tendency has been to increase mass time at the expense of quality time.

- The third stage is to face the challenge and to turn mass time into quality time and make the whole of higher education a quality experience.

This involves the application of appropriate learning-technology skills to the design of the syllabus, the course materials, and the assessment procedures. It takes on board the use of the most appropriate (not necessarily the sexiest) form of delivery.

It will always tempt the technocratic mind to think that technology will be able to do what people do, better than they do it already. Over the past two decades technology has been pushed forward as a way of replacing/reducing the role of the teacher. But materials based without good cause on a particular technology platform fail in two ways. First, like bad teachers they are inflexible, unadaptive and tiresome; secondly, they provide no exciting stimulus to trigger the creative interest of the student. Perhaps we all need to listen to a colleague of mine, Professor Steve Hepple, who often calls for us to begin 'delighting the student' with our courses.

So what is it that we should focus our creative abilities on that will make the most effective use of our time and talents, and that will 'delight' our students? I suggest that there are three 
broad issues we must attack quickly if we are to enter.into stage 3 of Wagner's list. These issues are:

- the role of the teacher in relationship to the student;

- course design and presentation;

- assessment models.

Clearly, these issues_are not totally separate, but lead on from one to the other with considerable overlaps in some cases. However, it is helpful to address them as steps in a process, and to apply an educational technologist's eye to them.

\section{The role of the teacher in relationship to the student}

In my paper 'Teaching and learning - a strategy for Anglia' (Harrison 1993), I suggest that 'a university education is a combination of complex and interacting learning experiences including, inter alia, formal lectures, tutorials and seminars, work based learning, resource materials, libraries and computer access, student support services, accommodation and the assessment procedures'.

The provision of an affordable experience for students depends on a careful analysis of requirements at discipline, course, institutional and employer levels. It must include an analysis of the potential and actual contributions of learning technology to teaching and learning in higher education. My own university is one that prides itself on the quality and expertise of its teaching and learning support staff. In many parts of the course structure, students have the opportunity for close contact with all these staff through tutorials, seminars, lectures and learning support facilities. As part of its continuing evaluation, Anglia Polytechnic, like all universities, is reviewing its teaching and learning methods. We believe that by allowing students more freedom to pace their study and by arranging modules with fewer rigidly fixed meeting points, they can organize their own learning more effectively. The development of credit accumulation and modular systems, a fundamental part of Anglia strategy, builds into our courses flexibility for both subject selection and timescales for completion of courses.

To achieve these changes, Anglia has developed new approaches to learning. In doing this, the university has cognizance of the interaction between teaching, the formal learning that stems from the organized structure of the planned teaching process, and the informal learning which occurs as a result of and which is encouraged by the flexible study methods and student-group interaction that flexible learning systems engender. This approach starts with the way the university clearly states the learning outcomes of undergraduate and postgraduate study, and of the individual modules, so that students can easily see what they have to achieve. Staff can use these outcomes to develop assessment methods to test the outcomes and competencies more accurately.

The university can also use this foundation to produce planned flexible study materials that will allow some parts of the course to be given to students early in their year so that they can organize their work-flow independently. Some of these materials will contain advice on group activities, and most will contain seminar and tutorial meetings that can be flexibly arranged by the students. At the same time, equal attention will be given to ensuring that the best practice associated with what has been called 'traditional' teaching is developed and incorporated into the strategy. 
The following represents the heart of the teacher's role:

Knowing:

- the subject;

- the students and their needs;

- the skills of presenting and managing the materials and processes of learning; and

- the best ways of assessing and providing feedback on the learning that is taking place.

Nothing in this suggests that teachers should never stand up in front of a class - nor does it suggest that the main function of a teacher is to stand up in front of a class. Rather, it says that the teacher is the best person to design the course, validate the information content, design the assessment, and work with the learners during the delivery phase. How the content is delivered must vary from module to module and course to course.

\section{Course design and preparation}

As part of this year's teaching and learning developments at Anglia, as Pro-Vice Chancellor (Teaching and Leaming) I have called for volunteers to suggest modules they believe can benefit from being presented in a more flexible form. I have no preconceived ideas about that form, though I expect that the most popular medium will be print on paper. From research I have recently undertaken, I have developed an approach to the structure of packaged materials that will, I believe, aid their rapid development, and ensure that we provide our students with a helpful consistency of approach.

However, I do not think that Anglia, or any university, can further impose upon the goodwill of its staff by expecting them to undertake the development of flexible materials and continue to carry their normal work loads. Because of this, I have recommended that monies be made available to buy out staff from normal duties to allow them safeguarded time to work with trained colleagues in the production of flexible learning materials.

\section{A new style for flexible learning packages.}

\section{The planning process}

Applying the techniques of learning technology to this process, one must first look at the content of the module and what one is trying to achieve from it. A number of decision points are discussed below that show that the package should be content-led rather than a decision being taken at the outset to base the package on a particular technology platform. The key decisions relate to the aims and learning outcomes of the course. Once these are in place, the best way of delivering them can be more reasonably assessed.

In using a particular delivery medium, it is important to consider the availability of the mechanism for 'playback' to the student who may be working at home or in some other remote location from the college where the package was designed.

Write, buy, adopt

Before jumping into developing new material, it is worth looking around for existing packages that can easily be adopted/adapted to meet our needs. Material from the Open Learning Foundation or the Open University are examples. 
Content - write or use textbook, etc.

A decision point is reached. Does one write the materials oneself and give readings that students can follow up? Does one build the module around a single book, video, film or whatever, tell the student to buy it, then direct students to use selected passages from it? Does one mostly write the material, give readings, and include in full some extracts that students might find difficult to find? Copyright regulations and necessary payments to rights holders must be considered. Therefore list the decisions:

- Set textbook, student to buy

- Recommended readings not included

- Selected reading to be included

- Video/audio/computer materials to be produced.

It will be important to talk to the library staff about stocking the necessary titles, and immediately to discuss media support with one's educational technologist.

\section{Module overview}

It will be necessary to prepare a brief statement that will later be used to guide the intending student in gaining an overview of the content. At this stage, it guides the later development of the module.

\section{Academic level}

Will the module be at introductory level, at a higher undergraduate level, or at a postgraduate level? The approach needs to be set from the start.

\section{Aims of the module}

Deciding on the aims of the module is a major task, and each aim should be large enough so that 10 or more learning outcomes can be derived from their content for trialling and assessment purposes. For example, learners should be able to:

- explain the notion of research design and how it relates to the decision problem;

- construct a questionnaire;

- do some important tests for examining the statistical significance of differences;

- carry out, using computer-based procedures, a statistical analysis of a reasonable data set.

Note that each of these aims must be able to sustain many pages of explanatory text and so forth, and provide material for assessment questions. These questions may, in the end, be selfassessed by the student, computer-assessed or tutor-assessed.

From each of the aims one will need to develop the appropriate learning outcomes. Each aim and its group of learning outcomes will then form a unit of study within the module.

\section{Learning outcomes}

Next, one takes the aims one by one, and one develops the appropriate number of outcomes to cover the achievement of each of them. It is often the case that 10 or so learning outcomes may be required to cover a single aim. The validity of an outcome will be tested by quickly attempting to devise a dozen or so True or False questions which, while they may never be used in the actual learning situation, will show if it is possible to test/assess completion and understanding of the outcome. The reasons for different assessment aims is discussed below. 


\section{Assessment models}

Atkins (1993) identifies several reasons for assessing students. As he puts it, they include:

- establishing the level of achievement reached at the end of a course or module;

- establishing progress during a course and to give feedback on it;

- diagnosing strengths and weaknesses leading to remedial action;

- consolidating work done so far - a learning experience in itself;

- motivating students;

- predicting a student's likely performance level in the future;

- determining whether a student is 'safe to practice';

- conforming to the requirements of external regulatory bodies;

- giving individual staff feedback on the effectiveness of their teaching;

- obtaining information on the effectiveness of the learning environment (and methods of presentation);

- monitoring standards over time.

These headings noted by Atkins provide a very useful guide to the range of questions one should plan to set so that various dimensions of the assessment can be met. Assessment can be built up in many ways, and to some extent our decisions will depend on how quickly we can provide a computer-based learning management system to underpin the use of flexible materials. However, three forms of assessment must first be considered.

First, there will be student-marked feedback questions designed to give assurance that the learners are on the right lines. These questions will test only the outcomes of one unit.

Secondly, there will be more complex multiple-choice questions that should be computergenerated and marked from a custom-designed databank. Initially, these may have to be marked by a tutor, or the students trusted to mark them themselves and to hand in the marked papers, or marked by a computer using a midway mark sensing card to collect answers. The questions may test integration of learning across two or more units of the module. Answers can be placed as an appendix to the module or issued later (though this creates additional work).

Thirdly, there will be assignments that draw content from the whole module or a major part of it, and that will test integration and understanding of the content or ability to perform skills. While these could be delivered by the computer, they will be tutor-marked. An examination may replace an assignment.

This summative and integrating assessment is an essential part of the learning process and should not be seen as 'just another assessment'. It will help 'make thinking and problem solving skills explicit, use holistic tasks, use occupationally relevant simulations' (Thinking and Learning at Work 1993). The more assessments integrate learning, the better the course and the more helpful the assessment. 'Learners constantly fed on a diet of "chopped up tasks" may sometimes never learn to chew or integrate all of the sub skills into one whole complex task.' (Thinking and Learning at Work 1993).

The volume of assignments must be related to the need for assuring the student and then ourselves that they have understood the content and, if appropriate, that they can perform the tasks. It should not be excessive and should be paced across the whole module. The weighting of assessments must be a matter of debate according to the subject. In designing the way assessment is treated in the packages, I confess that I lean towards a model of 'mastery' or . 
'competence'. By this, I mean that the student achieves an acceptable level of performance when tested against a set of predetermined criteria or standards. The activity can require intellectual, personal or practical achievements. They may be tested in a variety of ways. In Australia, I saw an excellent computer system that underpins the Deakin University flexiblelearning operations. It can be used just to assess, deliver, monitor, record (and so on) flexible modules, or to deliver and mark assessments for any or all students in the university. Known as the Computer Managed Learning (CML) package (Stubbs, 1993), it is a Vax/VMS-based system owned by CBTS and Campus America and costs in the order of $£ 15,000-£ 20,000$. It comes in two versions: the CML, which is the one used at Deakin University, and the CML/ CIS, a new release containing extra tools to help with the setting of questions at various levels of complexity. It apparently uses a version of Bloom's taxonomy to aid question design. It has the facility to control student progress through courses by releasing tests and assignments, to block progress if defaults are met, to update printed materials as students reach that part of the course, and to accept 'written' answers and pass them across to staff for on-screen marking and feedback. This is currently done via Vax Mail, but the latest release of the software has the facility built within the structure. Staff can then update student marks in real time. Computer-marked assessments are recorded automatically and students are allowed to pass to the next assessment point as soon as they are ready. It has a wide range of management information. This includes reports on screen or paper about the progress of groups, individuals, questions and how they are answered, cohorts, etc. All the staff who used the package were clear that it was rock solid, and they never had software problems. 135 users worldwide use CML, some 35 in Australia. Of those in Australia, three have more than 5,000 students using the system.

A tool like this is an essential part of supporting staff in their coping with the increasing volume of students that we seem destined to have on our campuses. I guess we could each write our own version, but frankly my advice is to join a club, be it this one or one of the others currently appearing in the market place.

\section{Building in the teacher}

Flexible learning does not imply that the students need be totally 'on their own', and tutorials can be built in as one deems appropriate. Students should have some choice of time and day, something which allows a package to have the teacher at the centre but also provides freedom of choice in attending a centre. Recent findings of what students enjoy most about higher education show that they like smaller group tutorials but can happily do without large-scale lectures. This facility could well be extended to distance students, with careful planning, and be delivered by telephone, electronic mail or video conference. There are well-qualified staff in regional colleges who can be the local expert to help students using a flexible package. Franchising of courses provides for this local support very well indeed.

These 'help' points need to be placed in the text of modules with details of how they will work and the location.

\section{Copyright}

It is vital to list all material to be reproduced within the learning package. An educational technologist will then be able to advise on any problems and costs, and will help get clearance for its use. Recent changes in the Copyright Licence mean that additional page charges apply if more than four copyright extracts are packaged together for five or more students. With a 
minimum charge of 8 pence per page per copy, this adds significantly to production costs (are publishers about to kill the goose with such high charges?).

\section{Evaluation}

Once designed and written, all modules will need to go through a peer-group evaluation process. Necessary revision can then be fed back into the information, design, and layout contents. The final stage will be the testing under user-conditions with even greater feedback being elicited from the students than one would usually seek. Flexible material, by its nature, needs to be open and accessible to students and to encourage them to return to the study. Therefore, the evaluation process must be more than just test results.

\section{Conclusion}

In summary, flexible packages offer many attractive advantages. They can retain the teacher at the centre of the experience. They offer greater choice not only of time, but place and pace of study. They offer a way of underpinning summer schools, since materials can be pre-issued to clients so that the short time they have is used to full advantage. They allow very rapid response to industrial needs. They build on the franchised regional links and its strengths. They provide real flexibility for students who cannot attend at fixed times for all sorts of reasons. Finally, they offer choice when numbers for a topic may be low.

\section{References}

Atkins, M.J. (1993), Assessment Issues in HES, London, Department of Employment.

Harrison, C. (1993), Teaching and Learning: A Strategy for Anglia, Cambridge, Anglia Polytechnic University.

Stubbs, N. (1993), 'Unmet demand and over-enrolment problems can be met with CML', paper delivered to Australian Universities, available from Noel Stubbs, CBTS (Australia) Pty Ltd., 62 Elgin Street, Carlton, Victoria, 3053 Australia.

Thinking and Learning at Work, Autumn 1993, Department of Employment.

Wagner, L. (1993), 'The economic and pedagogic imperative', Using Telematics in Education and Training, March. 